\title{
Psychiatric journals as the mirror of the dominant psychiatric model
}

\author{
Dusan Kecmanovic, ${ }^{1}$ Dusan Hadzi-Pavlovic ${ }^{2}$
}

The Psychiatrist (2010), 34, 172-176, doi: 10.1192/pb.bp.108.024018

${ }^{1}$ University of Belgrade, Serbia, and Newtown, Australia; ${ }^{2}$ University of New South Wales, and Prince of Wales Hospital, Randwick, Australia Correspondence to Dusan Kecmanovic (dkecmanovic@gmail.com)

\begin{abstract}
Aims and method Historical trends in the conceptual domains underlying articles published in psychiatric journals are indicators of major psychiatric concerns and practices. Articles in The American Journal of Psychiatry and The British Journal of Psychiatry during the periods 1947-51, 1967-71 and 2002-6 were classified into either a biomedical, psychological or social conceptual domain to determine which domains, if any, were dominant.
\end{abstract}

Results In The American Journal of Psychiatry one or two domains were dominant for two of the three periods. No domain was dominant in The British Journal of Psychiatry.

Clinical implications Examined against various scientific and social developments, American psychiatry appears more responsive to current social, scientific and commercial trends and impulses than British psychiatry.

Declaration of interest None.
The history of psychiatry reveals the waxing and waning of dominant conceptual models. These models (also called 'prominent emphases within psychiatry', 'predominant theoretical representations of psychiatry', 'approaches to the mind', ${ }^{3}$ and 'systems of explanations ${ }^{4}$ ) can be grouped into biomedical, psychological or social, as was done by Brodie \& Sabshin. ${ }^{5}$ We suggest that psychiatry has typically seen the dominance of one model, rarely two, while seemingly never have three models had the same relevance in the psychiatric community. In most cases, model shifts have been brought about by social and historical circumstances, rather than by any increased awareness among psychiatrists that a particular model better fits the nature of mental disorders.

Except for a short period of so-called moral treatment in the first half of the 19th century, the biomedical model dominated 19th-century psychiatry. In 1900, Freud's The Interpretation of Dreams marked the beginning of the affirmation of the psychodynamic model. In the 1950s and 1960s, cognitive-behavioural therapies were developed: these and the psychodynamic therapies are substantial parts of the psychological model.

Over the first half of the 20th century, while the psychological model gained ground, particularly in the USA, the relevance of the biomedical model did not decline and its central position was not threatened. These two models (and, correspondingly, 'biological psychiatry' and 'psychodynamic psychiatry') did not have anything in common; they coexisted, each with its own patients, vocabulary, explanatory principles, treatment methods and loci of treatment. From the mid-1960s, psychodynamic psychiatry started its still-continuing decline. ${ }^{6}$ In contrast, the cognitive-behavioural therapies have grown.

The 1960s saw the rise of the social model as the consequence of circumstances from both within and outside of psychiatry. The community mental health centre as the basic organisational unit for mental healthcare was introduced in the USA in 1963.

In the UK neither the psychological nor social model was as extensively endorsed as in the USA. The psychoanalytically-oriented Tavistock Clinic in London, for example, had limited influence on the British psychiatric community, ${ }^{7}$ while the social psychiatry innovation of the therapeutic community was confined to a small number of institutions: ${ }^{8}$ the social model was not institutionalised in the UK as it was in the USA.

The intellectual dominance of the social model did not last long, sidelined in the early 1980s by the reaffirmation of the biomedical model due to several factors. First, in the 1960s psychiatry was dominated by boundary expansion; then in the 1980s by boundary circumscription. ${ }^{1}$ Second, developments in genetics and neurobiology strengthened the belief that there is no psychiatry other than biological psychiatry. $^{9}$ Third, pharmaceutical companies, who had intruded into many aspects of psychiatric work, were emphatically interested in the re-medicalisation of psychiatry. ${ }^{410}$ Fourth, with many competitors for the care of people's minds, psychiatry needed to ensure its dominant position, which it attempted to do by adhering to a bioreductionist model of mental illness. Fifth, the Diagnostic and Statistical Manuals (from DSM-III onwards) placed psychiatry firmly back in the medical model. ${ }^{11}$ 
One way to assess whether a conceptual model dominates in a particular period is to examine the number of papers in psychiatric journals based on particular models. Journals can be used as primary research material because the papers published are the major product of research activity, lend themselves to breakdown by category ${ }^{12}$ and comprise objective and accessible data that make the results verifiable.

Patterns of publication are affected by a range of factors: editorial policy, the requirements of the associations which publish the journal (if relevant), and the number of submitted manuscripts pertaining to particular topics; the prevailing conceptual orientations too cannot but influence what types of texts are published.

Editorial policy and the dominant conceptual model are mutually dependent: the conceptual model exercises influence on the editorial policy, which mirrors and strengthens the model. In that sense, within a certain time span, the proportion of papers published showing the conceptual background of one of the models can be used as an indicator of that model's degree of dominance.

Although journals, especially nowadays, accept papers from all over the world, certain major journals still publish a good number of texts signed by researchers from the journal's country of origin and continue to mirror their own specific economic, social, political and other factors, not just global trends. So the proportion of papers based on a particular model in, for example, The American Journal of Psychiatry (AJP) and The British Journal of Psychiatry $(B J P)$, still gives a fair measure of the level of interest in that model in the USA and the UK.

We have identified three papers that have overviewed trends in research as reflected in psychiatric journals. Brodie \& Sabshin ${ }^{5}$ analysed papers published in the AJP and the Archives of General Psychiatry in the period 196372. The papers were first sorted according to whether their primary concern was with biological, psychological or social factors and then according to whether they focused on aetiology, treatment or intervening mechanisms.

Pincus et $a l^{12}$ surveyed those two journals for 1969-70, 1979-80 and 1989-90. They identified the field of research to which the article related (e.g. basic biological sciences, diagnosis/nosology, health/mental health services) and then classified it into 13 specific categories. In these two studies, only papers categorised as research articles were taken into account as the purpose was to describe the characteristics of psychiatric research.

Moncrieff \& Crawford $^{4}$ investigated change and continuity in the concerns and practices of psychiatry in Britain during the 20th century. Back issues of the BJP (Journal of Mental Science up to 1963) were reviewed for 1-year periods at the mid-point of each decade and each article was categorised in terms of its form (either research or commentary) and content.

Our goal was to assess whether there was any difference in the general conceptual approach of the articles published in two general psychiatric journals, the $A J P$ and the $B J P$, during the past 60 years. We chose to analyse those two journals because, as official journals of the American Psychiatric Association and the Royal College of Psychiatrists, they are the flagship publications of American and
British psychiatry, with a responsibility to publish from across the broad field of general psychiatry, much more so than specialised research journals or even the Archives of General Psychiatry. In this role they are leading psychiatric journals, having in 2007 the 3rd and 7th highest impact factors respectively for psychiatric journals.

We have not included journals from other countries, or other UK and US journals, because except for Annales Médico-Psychologiques in France and perhaps the Journal of Nervous and Mental Disease, there are virtually no journals from similarly sized countries, published across the relevant periods, which are likely to so fully reflect psychiatric thought and practice in their native countries.

\section{Method}

\section{Identification and categorisation of journal articles}

All articles classified as 'original articles' or 'papers' from three 5-year periods (1947-51, 1967-71 and 2002-6) were evaluated. Reviews and overviews, opinions, editorials, images in psychiatry, images in neuroscience, brief reports and case reports were excluded. Original articles, no matter how 'scientific', were regarded as the most accurate reflection not only of the major concerns and practices, but of the prevailing conceptual models in psychiatry.

Articles were classified according to which model constituted their conceptual background by examining the titles and abstracts (if any) and, if the title and abstract did not provide sufficient information, parts of the paper or the whole paper were read as well.

\section{Definitions of models}

Our definitions of basic psychiatric models are in accordance with those cited elsewhere. ${ }^{13-16}$

\section{Biomedical model}

This model represents mental disorder as an impairment of the biological constituents of the body, primarily of the brain, leading to a permanent or transitory loss of the capacity to perform typical activities of the body, primarily those of the brain, as well as to the appearance of distorted and/or atypical forms of mental functioning. Therapeutic measures relying on this model target the body/brain. Articles were identified as biomedical if they dealt with physical correlates or causes of mental disorders, effects of all forms of somatic treatments on the course and outcome of mental disorders, or biomedical concepts of mental disorders.

\section{Psychological model}

Within this model both psychic and somatic manifestations of mental disorders are construed as having a psychological background; psychological treatment methods are an integral part of this model. Articles falling under this model deal with: all aspects of psychological treatment; psychological causes of mental disorders; psychological concepts of mental illness; psychological measures; and description and/or analysis of particular psychological phenomena associated with individual mental disorders. 


\section{Social model}

Within this model mental disorders are explained as related to social factors and these factors also play an important, if not a key role, in the aetiological and therapeutic process. Here, articles dealt with: social correlates or causes of mental disorders; social therapies and their influence on the course and outcome of mental disorders; attitudes towards people with mental disorders; the protection of human rights of the mentally ill; legal aspects of the behaviour of mentally ill people; or the relationship between psychiatric staff and patients.

\section{Statistical analyses}

We used $\chi^{2}$ analysis for each journal at each period to test the null hypothesis that a third of articles fell into each domain. If that hypothesis was rejected, we tried to decide if one domain, or two, was dominant by comparing proportions. $^{17}$

\section{Criteria for determining dominance}

We were not aware of any existing criteria that could be applied in our analysis, therefore we devised them ourselves. For one domain to be dominant it had to either meet the A criterion 'domain was represented by more than $70 \%$ of articles' or the following B criteria:

1 domain represented less than $70 \%$ but more than $50 \%$ of articles

2 domain was significantly different from the next highest domain

3 difference between the other two domains was small (defined below).

For two domains to be dominant they needed to meet the $\mathrm{C}$ criteria:
1 domains' combined representation was more than $80 \%$ of articles

2 difference between the average of the two domains and the other domain had to be statistically significant

3 difference between the two domains was small.

The difference (\%) between two domains was converted into an effect size (ES) and the common definition of a small ES $(<0.2)$ was used as the criterion. ${ }^{18}$ These are conservative criteria that work against identifying a dominant domain (or domains) but we felt it was better to 'appear to miss' a finding than to identify one too easily. As the relevant percentages are straightforward, readers with a different formulation of dominance can readily decide whether it holds up in the data.

\section{Results}

Figure 1 shows the number of articles evaluated from each journal in each period, and the percentages falling in each domain. The hypothesis of equal proportions was rejected in all cases (with $P<0.0025$ ); criteria A, B and $\mathrm{C}$ were then applied as needed.

\section{American Journal of Psychiatry}

For the AJP the articles published in the 1947-51 largely confirm our hypothesis that one or two domains would be dominant in any given period. Of the classified articles, $46.5 \%$ had a biomedical, $43.5 \%$ a psychological, and $10.0 \%$ a social conceptual foundation; so according to the $\mathrm{C}$ criteria, biomedical and psychological domains were jointly dominant.

In the second period (1967-71), 35.9\% of articles had a biomedical, $13.4 \%$ psychological and $50.8 \%$ a social conceptual orientation. Although social articles were the

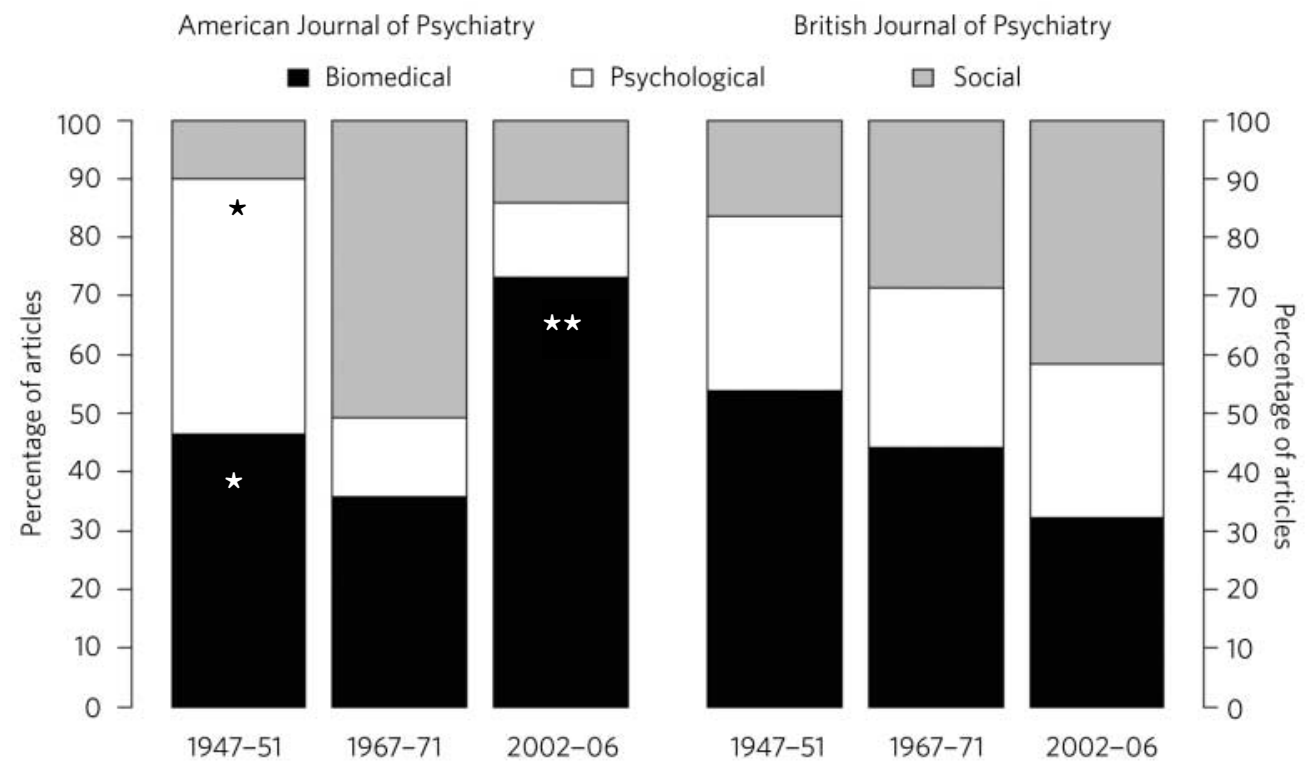

Fig 1 Number of articles evaluated and percentage of articles classified into each doman according to period examined and journal. ${ }^{\star}$, domains dominant according to criteria $C_{;}{ }^{\star \star}$, domains dominant according to criteria $\mathrm{A}$. 
most common (consistent with our hypothesis), they were not dominant according to our criteria.

Finally, in $2002-6,73.3 \%$ of articles had a biomedical conceptual foundation, $12.6 \%$ psychological and $14.1 \%$ a social one; according to the A criteria, biomedical domain was dominant, consistent with our hypothesis.

\section{British Journal of Psychiatry}

However, the results of the analyses of the papers published in the $B J P$ refute our hypothesis as in none of the periods could a domain be shown to be dominant. Of papers published in 1947-51, 53.8\% were biomedical, $29.8 \%$ psychological and $16.3 \%$ social. Across 1967-71, 44.0\% were biomedical, $27.3 \%$ psychological and $29.5 \%$ social. Finally, in the $2002-6$ period, $32.1 \%$ were biomedical, $26.3 \%$ psychological and $41.5 \%$ social.

\section{Foreign authorship of papers}

Not all articles are from a journal's country of origin, indeed the $B J P$ at one time actively supported authors from Commonwealth countries, which might be a factor influencing content. For 1 year from each period (1952, 1967 and 2002) we examined the proportion of the authors based outside the UK and the USA. In the BJP, the number of papers by researchers outside the UK in these years were 6 (12.6\%), $31(29.7 \%)$ and $27(38.0 \%)$ respectively. In the AJP, there were $12(11.7 \%), 4(3 \%)$, and 38 (27.5\%) articles published by foreign authors respectively.

\section{Discussion}

\section{Comparison with other studies}

It is hard to compare our results with previous studies $^{4,5,12}$ as periods and methods differ. We evaluated only those articles published as 'papers' or 'original articles', whereas other studies considered all articles in the main part of the journal ${ }^{4}$ or excluded articles they considered non-scientific., ${ }^{5,12}$

One study ${ }^{5}$ detected a decrease in biomedical and an increase in social papers in the AJP during 1963-72, but did not find social articles dominant in 1967-71.

When the nine scientific fields of research used in another study ${ }^{12}$ were grouped into our three categories for papers published in 1969-70 in the AJP, their results corresponded to ours. Out of the 68 scientific articles that could be classified, $32.4 \%(n=22)$ papers were biomedical, $8.8 \%(n=6)$ psychological and $58.8 \%(n=40)$ social. Our results for $1967-71$ were $35.9 \%, 13.4 \%$ and $50.7 \%$ respectively.

Where possible we grouped Moncrieff \& Crawford's $^{4} 15$ research fields into our categories. Out of 32 articles published in the BJP in 1945, 53.1\% ( $n=17)$ were biomedical, $34.4 \%(n=11)$ psychological and $12.5 \%(n=4)$ social. For 1947-51 we found $53.8 \%$ in the biomedical, $29.8 \%$ in the psychological and $16.3 \%$ in the social domain. Out of 96 articles from 1965, 38.5\% $(n=37)$ were biomedical, $35.4 \%$ $(n=34)$ psychological, and $26.0 \%(n=25)$ social papers. For $1967-71$ we found $44.0 \%$ biomedical, $27.3 \%$ psychological and $29.5 \%$ social papers.

\section{Analysis of findings}

Two findings deserve special attention. The first one pertains to the discordance of the number of biomedical, psychological and social articles published in the two journals in the three 5-year spans.

The proportions of biomedical and psychological articles published in the $A J P$ in 1947-51 are nearly equal (46.4\% and $43.35 \%$ respectively), whereas in the BJP biomedical articles (53.8\%) significantly outnumber psychological articles (29.8\%). This difference might be accounted for by the greater popularity of psychoanalysis among American psychiatrists than among their British counterparts in the period after the Second World War.

The 1967-71 period is one of growing trust in the power of pharmacotherapy and the heyday of social (community) psychiatry. The dominance of social articles (50.7\%) in the $A J P$ and the proportionally small number (29.5\%) of social articles published in the $B J P$ might be explained by the fact that, unlike in the UK, social (community) psychiatry was institutionalised in the USA. It had thus more sociopolitical repercussions, particularly as many regarded the community mental health centre programme as a logical extension of the fight for social justice. ${ }^{19}$ Issues novel to the protagonists of the mental healthcare delivery system, such as 'citizen involvement and consumer participation, accessibility of services and entitlement to care, community coordination and linkage, multidisciplinary professional relations, and the responsibility for "deinstitutionalized" populations ${ }^{, 19}$ were all themes discussed on the pages of the $A J P$ in the late 1960s.

Such a radical change in the psychiatric (and sociopolitical) landscape did not occur in Britain at that time, for 'While it has had some interests and involvement in community rehabilitation and psycho-social models of mental health, British psychiatry has not made any serious excursions as a profession into non-biomedical approaches analogous to... the community mental health movement of the 1960s in the US, ${ }^{20}$ Consequently, the pages of the BJP were not as 'saturated' by social articles.

The second finding deserving attention is a remarkable disproportion between the $A J P$ and the $B J P$ in the period 2002-6: nearly three-quarters (73.3\%) of articles published in the $A J P$ are biomedical articles $v$. a third (32.1\%) in the $B J P$ (in the same period the most numerous articles published in the BJP were social articles (41.5\%)). This disproportion might be construed in a number of ways.

First, modern research in biomedical psychiatry demands high levels of funding for laboratories and technology, requirements more easily met in the USA than either in the UK or the rest of the world. (It is worth noting though that American authors do not predominate in the $A J P$ just because of 'privileged' access.) Second, the $A J P$ has a much larger readership than the $B J P$, so that pharmaceutical companies, which financially support a substantial number of investigations of the efficacy of psychotropic medications (in particular key phase III trials), prefer to have the results published in the AJP. Third, the 'exceedingly' high number of biomedical papers in the $A J P$ in 2002-6 reflects the predominance of the biomedical or bio-bio-bio psychiatric model in current US psychiatry, ${ }^{10}$ 
a model that is more in tune with the spirit of the times in the USA than in the UK.

Eventually, as put by Samson, ${ }^{20}$ although there has been a clear shift of emphasis in American psychiatry since the mid-1970s towards the 'new biologism' and away from social and psychoanalytic models, few changes of theoretical directions appear to have affected British psychiatry. Or more accurately, even though mainstream psychiatry in the UK was mainly biological, 'this has not been to the exclusion of significant social and psychodynamic currents'. ${ }^{21}$ So much so in fact that, according to our results, not only has the proportion of psychological papers in the BJP remained more or less constant, but the other proportions have become transposed, a trend which, if it continues, could see the biological model usurped by the social.

Some caveats are appropriate. In both journals, although papers by native researchers outnumber those by foreigners, more papers by foreign researchers are being published than in the middle of the 20th century, the more so in the BJP. Therefore, although to some extent those journals might be reflecting international models, we suspect that it is more the case that foreign submissions reflect foreign readings of dominant, and hence publishable, models. Whatever the effect it is likely to be greater for the $B J P$ than the $A J P$ and makes our conclusions regarding the degree of concordance between trends in journals and trends in the UK and the USA less firm.

\section{Study limitations}

The primary data and conclusions are based on two general psychiatric journals, not research specific journals; nevertheless, we believe they were the right choice for our purposes. Our conservative definition of dominance might have understated differences. Finally, the papers were coded by only one person so no measure of reliability is available, but broad agreement with previous findings suggests that idiosyncratic ratings are unlikely.

\section{Conclusions}

Examining the results of our analysis against various scientific and social developments in the USA and the UK over the studied periods suggests that US psychiatry is more responsive to social, scientific and commercial trends and impulses than UK psychiatry, and shows strong trends of conceptual dominance explicable by concurrent social, scientific and professional concerns.

\section{Funding}

D.H.-P. was supported by the Australian Government National Health and Research Medical Council Program Grant no. 510135.

\section{About the authors}

Dusan Kecmanovic is visiting professor of psychiatry, University of Belgrade, Serbia, and a psychiatrist in Newtown, Australia, Dusan HadziPavlovic is conjoint senior lecturer, School of Psychiatry, University of New South Wales, and works as a senior hospital scientist at the Black Dog Institute, Prince of Wales Hospital, Randwick, Australia.

\section{References}

1 Sabshin M. Turning points in twentieth-century American psychiatry. Am J Psychiatry 1990; 147: 1267-74.

2 Beigel A. A proposed vision for psychiatry and the turn of the century. Compr Psychiatry 1995; 36: 31-9.

3 Havens LL. Approaches to the Mind: Movements of the Psychiatric Schools from Sects toward Science. Little Brown, 1973.

4 Moncrieff J, Crawford MJ. British psychiatry in the 20th century observations from a psychiatric journal. Soc Sci Med 2001; 53: 349-56.

5 Brodie HK, Sabshin M. An overview of trends in psychiatric research: 1963-1972. Am J Psychiatry 1973; 130: 1309-18.

6 Hale NG. The Rise and Crisis of Psychoanalysis in the United States: Freud and the Americans, 1917-1985. Oxford University Press, 1996.

7 Ramon S. Psychiatry in Britain: Meaning and Policy. Croom Helm, 1985.

8 Busfield J. Managing Madness. Hutchinson, 1986.

9 Guze S. Biological psychiatry: is there any other kind? Psychol Med 1989; 19: 315-23.

10 Sharfstein SS. Big Pharma and American psychiatry: the good, the bad, and the ugly. Psychiatr News 2005; 40: 3-4.

11 Blacker D, Tsuang MT. Classification and DSM-IV. In The Harvard Guide to Psychiatry (3rd edn) (ed AM Nicholi): 65-83. Belknap, 1999.

12 Pincus HA, Henderson B, Blackwood D, Dial T. Trends in research in two general psychiatric journals in 1969-1990: research on research. Am J Psychiatry 1993; 150: 135-42.

13 Lazare A. Hidden conceptual models in clinical psychiatry. N Engl J Med 1973; 288: 345-51.

14 Tyrer P, Steinberg D. Models for Mental Disorder: Conceptual Models in Psychiatry. John Wiley \& Sons, 1987.

15 Weckowicz TE. Models of Mental IIIness: Systems and Theories of Abnormal Psychology. Charles C. Thomas, 1984.

16 Siegler M, Osmond H. Models of Madness, Models of Medicine. Macmillan, 1974.

17 Goodman LA. On simultaneous confidence intervals for multinomia proportions. Technometrics 1965; 7: 247-54.

18 Cohen J. Statistical Power Analysis for the Behavioral Sciences (2nd edn). Lawrence Erlbaum, 1988

19 Brown BS, Goldstein $\mathrm{H}$. The lightning rod of human service delivery. In Controversy in Psychiatry (eds JP Brady, HK Brodie): 1041-2. WB Saunders, 1978.

20 Samson C. The fracturing of medical dominance in British psychiatry? Sociol Health IIIn 1995; 17: 245-68.

21 Crossley N. Contesting Psychiatry: Social Movements in Mental Health. Routledge, 2006. 